\section{Design, Implementation and Preliminary Testing of a Novel Orthosis for Reducing Erector Spinae Muscle Activity, and Improving Balance Control for Hyperkyphotic Elderly Subjects}

\author{
Veiskarami M. ${ }^{\oplus}$, Aminian G. ${ }^{2}$, Bahramizadeh M. ${ }^{2 *}{ }^{\circledR}$, Ebra- \\ himzadeh F. ${ }^{3}$, Arazpour M. ${ }^{2}$, Abdollahi I. ${ }^{4}$, Fadayevatan R. ${ }^{5}$
}

\begin{abstract}
Background: Aging often results in thoracic kyphosis and adverse postural changes. This may interfere with physiologic activity of paraspinal muscles. Few styles of spinal orthosis have been already used to reduce thoracic kyphosis. This paper describes the development of a novel orthosis, which is designed based on the anatomy of the back muscles. This novel orthosis may potentiate muscle activity and balance control among older hyper kyphotic subjects.

Objectives: The object of this study was to design and preliminary testing of a new orthosis to potentiate muscle activity and balance control among older hyper kyphotic subjects.

Material and Methods: In this quasi-experimental study, a new postural control orthosis with a textile band structure was designed to provide an additional support for spine and muscles of the back. The functional impact of this orthosis was evaluated in six older hyper-kyphotic subjects. According to the results, the paraspinal muscles activity and balance control were significantly improved.
\end{abstract}

Results: The RMS sEMG of the lumbar and thoracic erector spinae muscles reduced significantly $(\mathrm{p}<0.05)$, and a significant improvement $(\mathrm{p}<0.05)$ was observed in the balance test when patients put on the novel orthosis $(p<0.05)$.

Conclusion: The new orthosis can considerably improve the paraspinal muscles activity at both the upper and lower lumbar levels. It can also recover balance control among elderly subjects.

Citation: Veiskarami M, Aminian G, Bahramizadeh M, Ebrahimzadeh F, Arazpour M, Abdollahi I, Fadayevatan R. Design, Implementation and Preliminary Testing of a Novel Orthosis for Reducing Erector Spinae Muscle Activity, and Improving Balance Control for Hyperkyphotic Elderly Subjects. J Biomed Phys Eng. 2020;10(1):75-82. doi: 10.31661/jbpe.v0i0.1200.

\section{Keywords}

Kyphosis; Postural Balance; Paraspinal Muscles; Orthosis; Older Subjects

\section{Introduction}

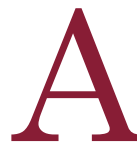

$\mathrm{s}$ human ages, the vertebrae undergoes continued deformities causing significant postural changes along the thoracic spine and this may create a shift in the trunk mass, an increased flexion deformity and excessive compression due to upcoming shearing forces to the spinal segments [1]. A posterior pelvic tilt may occur to provide a posterior adjustment to the vertebral position. The resulted tilt may subsequently cause either an unbalanced compensatory posture
${ }^{1}$ PhD Student, University of Social Welfare and

Rehabilitation Sciences,

Department of Orthotics

and Prosthetics, Tehran,

Iran

${ }^{2} \mathrm{PhD}$, University of

Social Welfare and

Rehabilitation Sciences,

Department of Orthotics

and Prosthetics, Tehran,

Iran

${ }^{3} \mathrm{PhD}$, Department

of Biostatistics and

Epidemiology, University

of Medical Sciences,

Lorestan, Khoramabad,

Iran

${ }^{4} \mathrm{PhD}$, University of

Social Welfare and

Rehabilitation Sciences,

Department of physi-

otherapy, Tehran, Iran

${ }^{5} \mathrm{MD}, \mathrm{MPH}, \mathrm{PhD}$, Univer-

sity of Social Welfare

and Rehabilitation Sci-

ences, Department of

Orthotics and Prosthet-

ics, Tehran, Iran

*Corresponding author:

M. Bahramizadeh

Department of Orthotics

and Prosthetics, Univer-

sity of Social Welfare

and Rehabilitation Sci-

ences, Tehran, Iran

E-mail: mbzoandp@

gmail.com

Received: 14 June 2019 Accepted: 3 August 2019 
or a compensated incongruent posture of the spine [2].This would change the sagittal plane alignment among the elderly and increase mechanical loading, which may compromise the strength of the extensor muscles of the back [3]. In addition, it interferes with normal function of the paraspinal musculature due to alterations in length-tension relationship, moment arm lengths, and force vector orientations [4].

The amount of abnormal spontaneous activity of the para-spinal muscles increases with age at both upper and lower lumbar levels [5]. This explains the pathology of kyphosis and suggests that reduction in muscle activity might serve as an index for treatment options [5]. Considering this association, it might have positive impacts on vertebral loading, trunk control, and balance, which in turn reduces the risk for fractures.

There have been a number of therapeutic mythologies such as postural taping, postural training and the use of spinal orthosis to resolve the above-mentioned issue. However, there are limitations for each one [6-8], for example, as orthosis causes limited pelvic rotations, walking speed reduces [9]. The new generation of conventional orthosis also limits hip function [10].

In fact, none of the previous devices was designed based on the anatomy of the regional muscles to reduce muscle activity for elderly hyperkyphotic subjects. This new orthosis may even improve the muscle activities among older people. Since previous studies have shown this postural changes might be associated with a relatively lower gait speed and balance control [11], we have also investigated the impact of our intervention on walking speed and balance control as well.

\section{Material and Methods}

\section{Design Considerations}

In this quasi-experimental study, preliminary study of vertebral column and posterior back muscles revealed that the anatomical el- ements display a columnar muscular design, which interchanged with a band-like structure. Therefore, we proposed bands of textile materials to design the orthosis.

\section{Orthosis Elements Design}

Since weakness of the back muscles has likely caused the abnormal spinal curvature, muscles of dorsum as well as the para-vertebral muscles were targeted to be studied as an inspiration for the elements of our recommended orthosis. Few individual muscles such as erector spinae, trapezius, serratus posterior inferior, latissimus dorsi and internal oblique were selected for investigation because they play a significant role in supporting the back [12].

\section{Band placements}

The structure of our orthosis includes a central vertical portion and three bands in other directions. The bands are adjustable based on the height of spinal column. To balance the spinal posture, the vertical part of the orthosis is aligned with vertebral column in order to work as an additional column to protect the central part of the orthosis all over the thoracic and lumbar regions. This may also provide further support to the erector spinae muscles and potentially lessens the abnormal curvature of the spine.

The orthosis bands actually follow the same directions and attachment of the back muscles in order to control thoracic kyphosis and lumbar lordosis. The thoracic band originates bilaterally from the spinous process of T6 over the muscle bulk of trapezius toward the anterior aspect of the acromioclavicular joints. The dynamic force of elastic bands pulls the shoulders back in order to correct the kyphosis [8]. In thoraco-lumbar region (T11-T12), there is an X-shape band to provide an additional support to serratus posterior inferior and latissimus dorsi muscles. The bands cross at this anatomical point (T11-T12) and provide double support for the spinal curvature at thoracic 
A Novel Orthosis for Hyperkyphotic Elderly Subjects

and lumbar levels [13].

Since the erector spinae muscle is attached to sacrum and pelvis, it may increase lumbar lordosis by causing an anterior tilt to the pelvis. In addition, the upper anterior fibers of internal oblique muscle tilts the pelvis to the front when thorax is fixed [14]. Therefore, they both support and stabilize the sacroiliac joints [15]. That is the reason the orthosis bands are applied on them so that they help to promote tilting the pelvis forwardly.

The central part of the orthosis originates

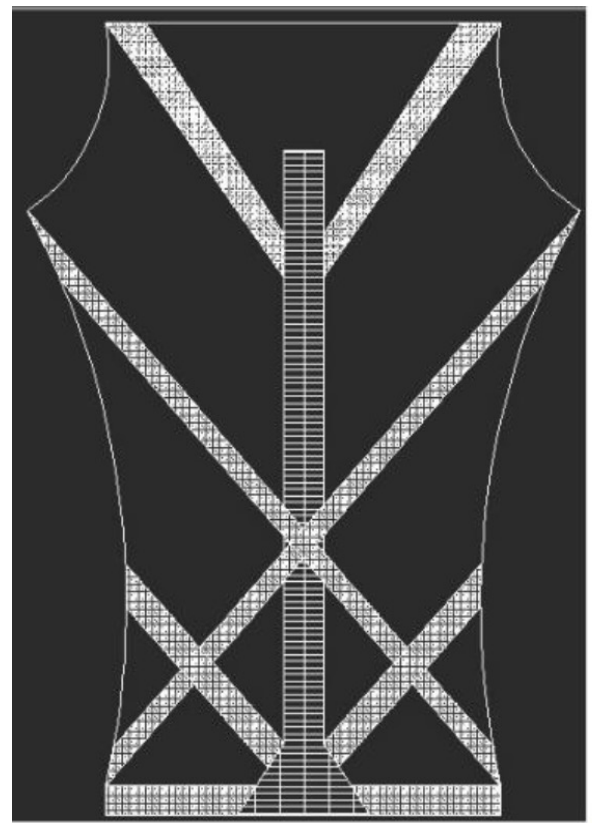

from iliac crest in direction of erector spinae muscles to impose a mechanical effect on anterior pelvic tilt (Figure 1). In order to potentiate the activity of internal oblique muscle and impose a mechanical effect, a band was applied in sloping direction. It starts from the middle one-third of the intermediate line of the iliac crest to the inferior borders of $10-12^{\text {th }}$ ribs, where linea alba is located. There is another band in direction of PSIS to ASIS in order to limit posterior pelvic tilt while it potentiates the stability of the sacroiliac joint.

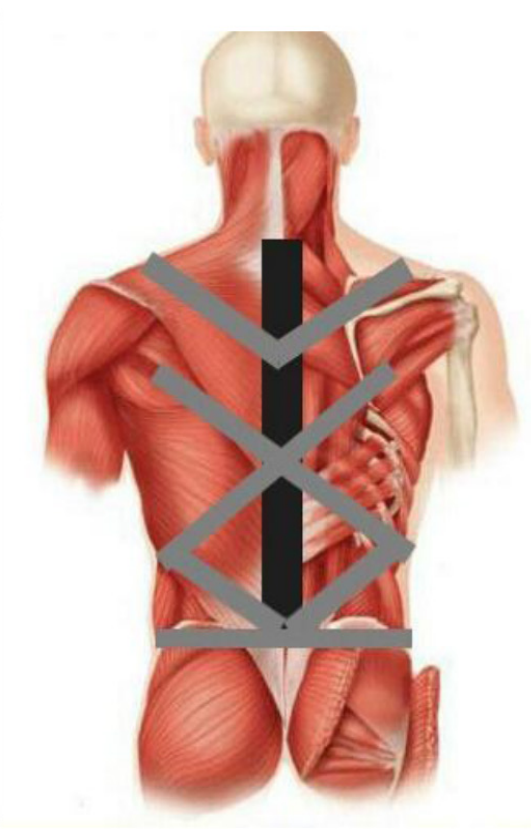

Figure 1: Schematic design of new spinal orthosis

\section{Band Materials}

The central part of the orthosis contains bands of cotton neoprene as well as bands with light breathable polyester stretch. This will support the central column more efficiently. Soft cotton covers the adjustable elastic bands protected in order to avoid pinching and binding because the vertical band has to have more resistance than the outer band.

\section{Testing of Intervention}

Six older healthy subjects (mean age:
69.7 \pm 2.98 years, mean height: $157.5 \pm 2 \mathrm{~cm}$, and mean weight: $63.5 \pm 5.44 \mathrm{~kg}$ ) with a thoracic kyphosis greater than 40 degrees were selected for the study (Table 1). People, who were registered in the project as participants, were excluded from the study if they had a history of lower extremity or spinal surgery, vertebral fractures, spondylolysis, spondylolisthesis, congenital abnormalities of the spine, disc herniation, visual or vestibular impairment, diabetes, neurological dysfunction or history of taking any medicine interfering 
Table 1: The mean and standard deviation (SD) for participants.

\begin{tabular}{cc} 
Subject's Characteris-tics & Mean and SD \\
\hline Height(cm) & $157.5 \pm 2$ \\
\hline Weight(kg) & $63.5 \pm 5.44$ \\
\hline Age(years) & $69.7 \pm 2.98$ \\
\hline Kyphosis(deg.) & $48.75 \pm 2.21$
\end{tabular}

with balance control. The Ethical Committee of the University of Social Welfare and Rehabilitation Sciences approved the study.

Prior to the study, the thoracic kyphosis was measured using Dualer Electric Inclinometer, North American Fork, Utah. An experienced investigator was assigned to manage the intervention according to the instruction manual [8]. Functional tests and electromyography of the muscles were measured before starting the study. We repeated those tests after a patient put on the new prototype orthosis and carried it out for at least 10 minutes.

Electromyography was done for lumbar and thoracic erector spinae muscles on both sides with a portable electromyogram (ME3000P, Mega Electronics Ltd., Finland) in resting as well as standing positions for a minute [5]. Patients were prepared for an EMG tests. The skin of the lower back was cleaned and shaved. The surface electrodes were placed over thoracic erector spinae at a point $5 \mathrm{~cm}$ lateral to T9 spinous process, and lumbar erector spinae muscles at a point $3 \mathrm{~cm}$ lateral to $\mathrm{L} 4$ spinous process. Each pair of electrodes was spaced $3 \mathrm{~cm}$ center-to-center along the directions of the muscle fibers. A reference electrode was placed laterally over the right $10^{\text {th }}$ rib (Figure 2). Timed Up and Go Test (TUG) and the 6-meter walking speed (6MTW) tests were selected to investigate the performance of the orthosis on dynamic balance and gait speed.

Finally, the EMG data were analyzed using MegaWin (Mega Electronics Ltd.) software. The EMG raw data was analyzed using root mean square (RMS) to obtain the average amplitude of the EMG signal. The non-parametric Wilcoxon test was applied to identify whether significant differences existed before and after the intervention, and the results are therefore presented as the median and interquartile

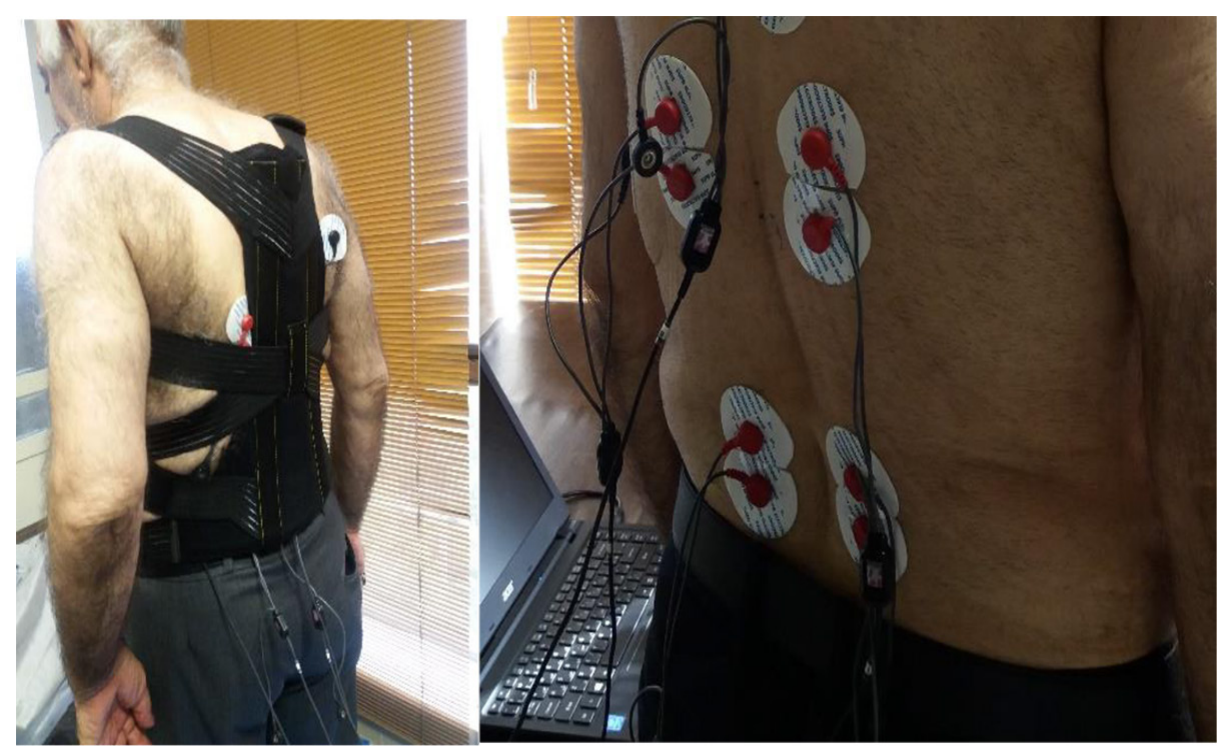

Figure 2: A subject put on the orthosis and the EMG markers were placed. 
range. A value of $\mathrm{P}<0.05$ was considered as indicator of statistical significance.

\section{Results}

The RMS values of each muscle with and without orthosis are presented in Table 2.

The RMS sEMG of the lumbar at thoracic erector spinae muscles reduced significantly after wearing the novel orthosis $(\mathrm{p}<0.05)$. Likewise, A significant improvement $(p<0.05)$ was observed in the TUG test Score when patient put on the orthosis $(\mathrm{p}<0.05)$ (Table 3$)$. Although it did not reach the significant level, walking speed decreased when the orthosis was used.

\section{Discussion}

In this study, we have presented a novel spinal orthosis, which is designed to support the erector spinae muscle and improve balance control among the older hyperkyphotic subjects. This orthosis will likely improve the patient's functional class in many aspects. The functional and electromyography activity tests indicate a significant improvement in balance control and muscle activity after wearing our postural control orthosis.

The other novel orthosis was previously investigated; however, results obtained in this study may not be consistent with the other results. There are only three previous studies available in respect to the performance of an orthosis on erector spinae function with their own methodologies [9, 16, 17]. Unlike the conventional orthosis, there is no rigid elements in this study to prevent muscle activity; therefore, it causes upper trunk extension, which may reduce lower back exten-

Table 2: Median and interquartile range of RMS values of trunk muscles with and without orthosis (w/o).

\begin{tabular}{|c|c|c|c|c|c|c|}
\hline \multirow{3}{*}{ Muscle } & \multirow{3}{*}{ side } & \multicolumn{4}{|c|}{ RMS value } & \multirow{3}{*}{$P$ value } \\
\hline & & \multicolumn{2}{|c|}{ W/O orthosis } & \multicolumn{2}{|c|}{ With orthosis } & \\
\hline & & Median & $\begin{array}{l}\text { Interquartile } \\
\text { range }\end{array}$ & Median & $\begin{array}{l}\text { Interquartile } \\
\text { range }\end{array}$ & \\
\hline \multirow{2}{*}{$\begin{array}{c}\text { Thoracic erector } \\
\text { spinae }\end{array}$} & Right & 40.5000 & 5 & 36.0000 & 4 & 0.011 \\
\hline & Left & 38.5000 & 4 & 34.5000 & 6 & 0.011 \\
\hline \multirow{2}{*}{$\begin{array}{l}\text { Lumbar erector } \\
\text { spinae }\end{array}$} & Right & 40.00000 & 7.25 & 32.5000 & 7 & 0.012 \\
\hline & Left & 39.0000 & 4 & 34.0000 & 6 & 0.011 \\
\hline
\end{tabular}

Table 3: Median and interquartile range of TUG and 6-MWS test with and without orthosis.

\begin{tabular}{cccccc}
\multirow{2}{*}{ Condition } & \multicolumn{2}{c}{ W/O orthosis } & \multicolumn{2}{c}{ W/O orthosis } & P-value \\
\cline { 2 - 5 } & Median & Interquartile range & Median & Interquartile range & \\
\hline TUG scor(s) & 17.5000 & 3.1 & 15.7000 & 3.2 & 0.008 \\
\hline 6-MWS score(s) & 10.0000 & 2.2 & 9.0000 & 2.7 & 0.08
\end{tabular}


sion moments. Likewise, our orthosis causes a significant force to apply into the posterior pelvic area in order to control lordosis, which protects posterior muscles of the pelvis. The double advantages of the orthosis would largely improve the malalignment seen among the elderly population; thus, it reduces the rate of falls and lower back pain [18].

This orthosis works similar to a few other ones such as the orthosis with joints providing resistive force (ORF), and rucksack-type and spinomed orthosis. Both ORF and rucksack-type orthosis can hold a relatively large magnitude of force applied to the upper trunk and reduce erector spinae activity. However, the ORF orthosis limits pelvic rotation as a major determinant of normal gait, which results in reduced walking speed [19]. Likewise, rucksack-type orthosis increases gravity to the upper trunk; however, it causes compression to the lower back area in proportion based on weight [9]. The pelvic straps of spinomed orthosis are placed under the hip. This may cause a resisted range of motion of hip joint and consequently a limited hip function [10].

The novel orthosis provides a relatively higher performance compared to the previous ones. In fact, there are two different mechanisms for this advantage. First, the device produces a considerable force over the shoulders and reduces anterior compressive force exerted on a kyphotic spine. This would maintain the center of gravity within the base of support. Second, the elements of our orthosis may increase proprioceptive input and enhance the patient's ability to sense the position of the spine. This would increase the back's extensor muscles control. It also reduces the electrical activity of the muscles and painful contractions of the erector spinae muscles. Moreover, these effects decrease the overall load over the anterior aspect of the spinal column and potentiate dynamic postural stability.

During the preliminary testing, the resulted balance control shortened the length of time for Up and Go Test (TUG) when the sub- ject put on the orthosis. In fact, the TUG test measures components such as sit-to-stand, gait, and turning skills required for postural control. These skills are extremely important because many falls occur during ambulation [20]. Likewise, the 6- meter walking test not only showed no negative impact on walking speed but also potentiated walking velocity although not significantly. This can be due to an improved balance when patient put on this orthosis.

\section{Conclusion}

The results of the current study show that this novel orthosis can reduce back extensor muscles activity and improve balance control in older hyperkyphotic individuals. Our study suggests that the elderly with a hyperkyphosis posture might be able to stand and walk more efficiently when wearing this novel orthosis. This orthosis is low-cost and can be easily applied into daily living. We believe this orthosis can work as a physiotherapeutic index for hyperkyphotic elderly subjects. Further studies with a larger sample size are warranted to provide better conclusive results based on the efficacy of this novel orthosis among older hyperkyphotic subjects to demonstrate the static and dynamic performance of the orthosis.

\section{Acknowledgment}

The authors thank Lorestan Physical Education Department and Sedigh Senior Care Center for assistance for data collection.

\section{Conflict of Interest}

None

\section{References}

1. Briggs AM, Van Dieen JH, Wrigley TV, Greig AM, Phillips B, Lo SK, et al. Thoracic kyphosis affects spinal loads and trunk muscle force. Phys Ther. 2007;87:595-607. doi: 10.2522/ ptj.20060119. PubMed PMID: 17472956.

2. Knight $R$, Jackson R, Killian J, Stanley $E$, Lowe T, Winter R. White paper on sagittal plane alignment. Terminology Committee of 
A Novel Orthosis for Hyperkyphotic Elderly Subjects

Scoliosis Research Society. 2003.

3. Mika A, Unnithan VB, Mika P. Differences in thoracic kyphosis and in back muscle strength in women with bone loss due to osteoporosis. Spine (Phila Pa 1976). 2005;30:241-6. doi: $\quad 10.1097 / 01 . b r s .0000150521 .10071 . d f$. PubMed PMID: 15644764.

4. McGill SM, Hughson RL, Parks K. Changes in lumbar lordosis modify the role of the extensor muscles. Clin Biomech (Bristol, Avon). 2000;15:777-80. PubMed PMID: 11050362.

5. Enomoto M, Ukegawa D, Sakaki K, Tomizawa $S$, Arai $Y$, Kawabata S, et al. Increase in paravertebral muscle activity in lumbar kyphosis patients by surface electromyography compared with lumbar spinal canal stenosis patients and healthy volunteers. J Spinal Disord Tech. 2012;25:E167-73. doi: 10.1097/ BSD.0b013e31825d63c4. PubMed PMID: 22614265.

6. Kaplan RS, Sinaki M. Posture Training Support: preliminary report on a series of patients with diminished symptomatic complications of osteoporosis. Mayo Clin Proc. 1993;68:11716. doi: 10.1016/s0025-6196(12)60067-6. PubMed PMID: 8246618.

7. Sinaki M, Lynn SG. Reducing the risk of falls through proprioceptive dynamic posture training in osteoporotic women with kyphotic posturing: a randomized pilot study. Am J Phys Med Rehabil. 2002;81:241-6. doi: $\quad 10.1097 / 00002060-200204000-00001$. PubMed PMID: 11953540.

8. Greig AM, Bennell KL, Briggs AM, Hodges PW. Postural taping decreases thoracic kyphosis but does not influence trunk muscle electromyographic activity or balance in women with osteoporosis. Man Ther. 2008;13:249-57. doi: 10.1016/j.math.2007.01.011. PubMed PMID: 17433756.

9. Katsuhira J, Matsudaira K, Oka H, lijima S, Ito $A$, Yasui $T$, et al. Efficacy of a trunk orthosis with joints providing resistive force on low back load during level walking in elderly persons. Clin Interv Aging. 2016;11:158997. doi: 10.2147/CIA.S108033. PubMed PMID: 27877028. PubMed PMCID: PMCPMC5108480.

10.Azadinia F, Kamyab M, Behtash H, Maroufi N, Larijani $B$. The effects of two spinal orthoses on balance in elderly people with thoracic ky- phosis. Prosthet Orthot Int. 2013;37:404-10. doi: $10.1177 / 0309364612474487$. PubMed PMID: 23401294.

11.Kado DM, Huang MH, Nguyen CB, BarrettConnor E, Greendale GA. Hyperkyphotic posture and risk of injurious falls in older persons: the Rancho Bernardo Study. J Gerontol A Biol Sci Med Sci. 2007;62:652-7. doi: 10.1093/ gerona/62.6.652. PubMed PMID: 17595423.

12.Saladin K, McFarland R. Human anatomy. 2nd ed. New York: McGraw-Hill; 2008.

13.Lyu S [Internet]. Posture Modification Effects Using Soft Materials Structures. Retrieved from the University of Minnesota Digital Conservancy. Avilable from: https:// conservancy.umn.edu/bitstream/handle/11299/182322/Lyu_umn_0130E_17306. pdf? sequence $=1$ \&isAllowed $=\mathrm{y}$.

14.Kendall FP, McCreary EK, Provance PG. Muscles: testing and function with posture and pain. Philadelphia: Lippincott Williams \& Wilkins; 2005.

15.Neumann DA. Kinesiology of the musculoskeletal system-e-book: foundations for rehabilitation. New York: Elsevier Health Sciences; 2013.

16. Ishida $\mathrm{H}$, Watanabe $\mathrm{S}$, Yanagawa $\mathrm{H}$, Kawasaki $M$, Kobayashi Y, Amano Y. Immediate effects of a rucksack type orthosis on the elderly with decreased lumbar lordosis during standing and walking. Electromyogr Clin Neurophysiol. 2008;48:53-61. PubMed PMID: 18338535.

17. Katsuhira J, Matsudaira K, Yasui T, lijima S, Ito A. Efficacy of a trunk orthosis with joints providing resistive force on low-back load in elderly persons during static standing. Clin Interv Aging. 2015;10:1413-20. doi: 10.2147/ CIA.S85294. PubMed PMID: 26366062. PubMed PMCID: PMCPMC4562746.

18. Katsuhira J, Matsudaira K, Oka H, lijima S, Ito $A$, Yasui $T$, et al. Efficacy of a trunk orthosis with joints providing resistive force on low back load during level walking in elderly persons. Clin Interv Aging. 2016;11:158997. doi: 10.2147/CIA.S108033. PubMed PMID: 27877028. PubMed PMCID: PMCPMC5108480.

19. Inman VT, Eberhart HD. The major determinants in normal and pathological gait. JBJS. 1953;35:543-58.

20.Thomas JI, Lane JV. A pilot study to explore 
Veiskarami M., Aminian Gh., Bahramizadeh M. et al

the predictive validity of 4 measures of falls risk in frail elderly patients. Arch Phys Med

Rehabil. 2005;86:1636-40. doi: 10.1016/j. apmr.2005.03.004. PubMed PMID: 16084819. 